\title{
Microlens array-based high-gain screen design for direct projection head-up displays
}

\author{
M. Kivanc Hedili, ${ }^{1}$ Mark O. Freeman, ${ }^{2}$ and Hakan Urey ${ }^{1, *}$ \\ ${ }^{1}$ Optical Microsystems Laboratory, Koc University, Rumelifeneri Yolu, Sarıyer, İstanbul 34450, Turkey \\ ${ }^{2}$ Lost Lake Technology LLC, 21623 W. Lost Lake Rd., Snohomish, Washington 98296, USA \\ ${ }^{*}$ Corresponding author: hurey@ku.edu.tr
}

Received 2 October 2012; revised 28 December 2012; accepted 29 December 2012; posted 18 January 2013 (Doc. ID 177329); published 20 February 2013

\begin{abstract}
Head-up display (HUD) systems have been used in recent car models to provide essential information to the drivers while keeping their eyes on the road. Virtual image HUD systems have been the preferred method, but they have the drawback of requiring a large volume of space in order to accommodate the relay optics that creates the virtual image. This is especially significant as the desired field of view increases. Direct projection HUD systems have been developed with a separate stand-alone microlens array (MLA)-based transparent screen on the dashboard, offering a compact solution. In this paper, we propose a direct projection HUD system based on a unique, windshield-embedded see-through screen that uses minimal space under the dashboard, offering an elegant and compact solution to the HUD problem. The screen is based on MLAs with varying surface normal angles such that the light from the projector is directed to the viewer's eyes from all positions across the field of view. Varying tilts provide an efficient relay and high brightness even with a low-lumen output projector. The calculated screen gain is about 69 and the eyebox area is about $30 \mathrm{~cm} \times 30 \mathrm{~cm}$. (C) 2013 Optical Society of America

OCIS codes: $\quad 120.2820,240.3990,080.4295,080.2740$.
\end{abstract}

\section{Introduction}

Modern cars have many displays at different locations, which can divert the driver's attention. Headup displays (HUDs) reduce the risk of accidents by keeping the driver's eyes on the road [1-3]. As a result HUDs are becoming popular among new car models. Virtual image HUD systems occupy a large volume of space under the dashboard since the optical relay and the eyebox expander components are large and need considerable distance between them [4]. The eyebox expanders are essential to increase the area in front of the viewer from which the display information can be observed.

Direct projection HUD systems, on the other hand, form a real image on a special HUD screen, located close to the windscreen. The screen serves as the eyebox expander without requiring additional relay

$1559-128 X / 13 / 061351-07 \$ 15.00 / 0$

(C) 2013 Optical Society of America optics from the projector to the eye [5]. The screen needs to angularly expand the reflected light to form an eyebox while providing high-transmittance seethrough capability much like a piece of glass. We recently published a direct projection HUD system using a commercial picoprojector and a custom-made screen, as seen in Fig. 1. Because of the unique fabrication method used, the screen does not distort the clear view of the surrounding area that is seen through the screen, while the projected image or video content is displayed [5].

The most important advantages of the direct projection HUD compared to the virtual image HUD are (i) scalability to much larger screen sizes [and field of view (FOV)] without increasing the overall size of the system as the size of relay optics and complexity increase rapidly with increasing FOV in virtual image HUD systems; (ii) portability, as it does not require installations that need custom-designed lenses; and (iii) lower cost due to fewer components required. The main disadvantage is that the drivers need to 


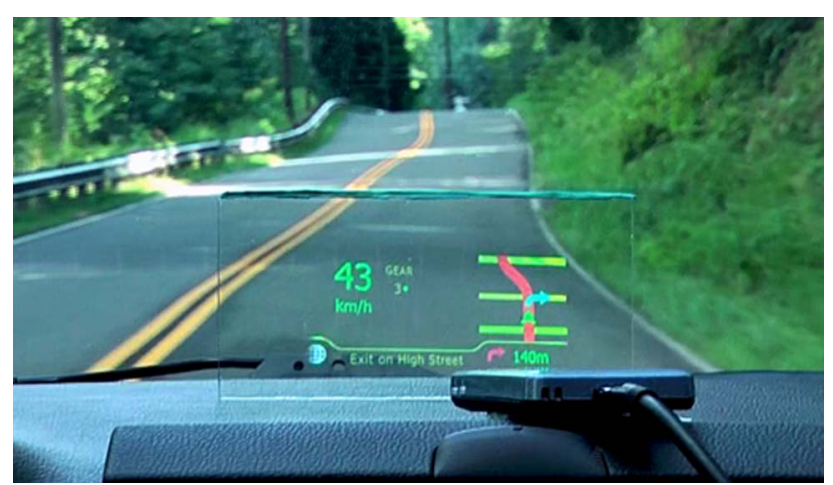

Fig. 1. (Color online) Real photograph of direct-projection HUD in operation. The unique structure of the screen provides good reflectance for the projected image coupled with very good transmittance. In this figure, the screen is not embedded into the windshield.

adjust their focus to see the content while driving [5]. While this is an important property offered by the virtual-image HUDs, the direct projection HUDs still offer a significant safety advantage by keeping the driver's eyes on the road. Also, one could envision a future display where a virtual image HUD is used for a portion of the display and a direct projection HUD for the majority of the field of view.

In this paper, we present a new direct projection HUD system and design methodology where the transparent screen is embedded into the windshield and a projector is incorporated into the dashboard. The new screen design is based on a partially reflective microlens array (MLA) where the axis of each microlens is tilted such that the reflected light is directed toward the driver's eyes. For a built-in system, this offers an elegant HUD solution that has negligible impact on the car's interior design.

In Section 2, we introduce the see-through screen structure, its operation principle, and a possible system geometry. In Section $\underline{3}$, the method for calculating the rotation angles is explained, and in Section $\underline{4}$, simulation results are discussed.

\section{Direct Projection HUD Design}

The key component of our direct projection HUD system is the embedded see-through screen. MLA-based eyebox (or exit pupil) expanders have been successfully demonstrated with color projectors [4-6]. In a planar reflective MLA, the central direction of the reflected light is governed by the usual law of reflection, i.e., angle of incidence equals angle of reflection. The exit pupil or the eyebox is expanded about this central direction due to the curvature of the microlenses. The term "eyebox" refers to the area in front of the driver's eyes from which the HUD information can be observed. It is analogous to looking through a window suspended in space. Since the incident light is at a slightly different angle for each pixel on the screen, the eyebox generated by each individual pixel is shifted by a different amount at the driver's position. Consequently, the full content on the screen can only be viewed from the overlapping region of all of

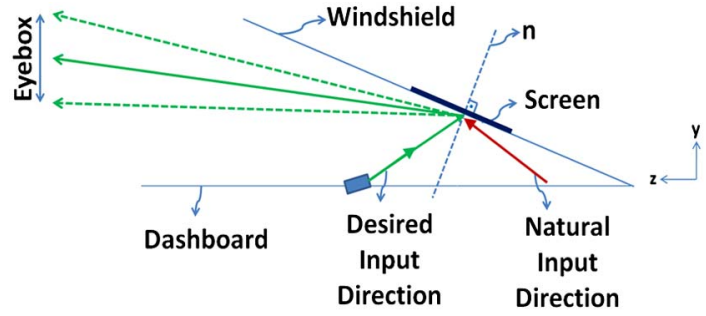

Fig. 2. (Color online) Because of the angle of the windshield, the natural input direction is behind the screen. The desired input direction is closer to the driver so that the geometric distortions are minimized and the projector has adequate distance to project onto the whole screen.

the individual eyeboxes, which is smaller than the individual eyeboxes themselves.

The natural input direction for the windshield embedded see-through screen, that is, the incident beam direction that would relay the light to the driver's eye, is shown in Fig. 2. Both the grazing angle of incidence onto the screen and the short projection distance available in most cars from this position are problematic. Projecting on an inclined surface causes keystone distortion but that can be compensated electronically in the video channel of the projector. The short projection distance results in too small an image to be of practical use for an automotive HUD. The easiest way to increase the projection distance is to place the projector closer to the driver's side, as indicated by the "desired input direction" in Fig. 2. Moving from the natural input direction toward the desired input direction increases the shadowing effect, which will be discussed later; consequently, there is a trade-off between the two input positions.

More importantly, the inclination of the screen results in an increased shift in each pixel's eyebox position. To illustrate this, we have simulated the four corners and the center of the planar MLA screen projected from the natural input direction for the geometry in Fig. 2. The resulting eyeboxes have no overlapping region, as seen in Fig. 3 . This means that with the windshield-embedded planar MLA there is

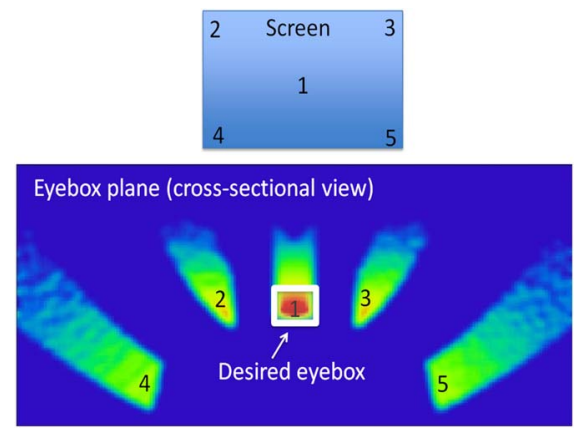

Fig. 3. (Color online) Simulated eyeboxes corresponding to the five points across the planar MLA screen that is illuminated from the natural input direction. This approach does not work, as there is no overlapping region of the individual eyeboxes so the full content on the screen cannot be seen from any position. 
no viewing position from which the driver would be able to view the entire display. In addition, each of the single pixel eyeboxes exhibits severe geometric distortion. Solving this problem is the subject addressed in this paper.

\section{A. System Layout}

Since the rotation angles of the microlenses are dependent on the system geometry, such as the positions of the driver, the screen, and the projector, we propose a design that we believe is suitable for a wide range of automobiles. In our design, we assumed that the angle between the $z$ axis and the windshield is $34^{\circ}$. The screen size is $175 \times 87.5 \mathrm{~mm}$ and the height of the center of the screen is $81 \mathrm{~mm}$ from the dashboard. The driver is $1200 \mathrm{~mm}$ away from the bottom of the windshield and the eyes are $250 \mathrm{~mm}$ above the dashboard. The eyebox, which is centered on the driver's head, has a shape and size determined by the shape of the microlens aperture and the radius of curvature, respectively. We used rectangular microlenses to produce a rectangular shaped eyebox. The $3.2 \mathrm{~mm}$ radius of curvature of the microlenses has been optimized using Zemax to yield the desired eyebox size of about $30 \mathrm{~cm} \times$ $30 \mathrm{~cm}$ at the driver's position.

With the specifications above, the proposed design is illustrated in Fig. 4. The projector is placed on the dashboard with an angle to illuminate the screen. Alternatively, the projector could be buried within the dashboard since the projector engines are quite small for modern picoprojectors. It can be electrically connected to the vehicle to receive the necessary information to be displayed. If there is not enough distance for the projector to illuminate the entire screen, the projection cone can be expanded by a small lens in front of the projector without adding much to the overall system size.

Note that the design methodology introduced in the paper works for arbitrary placement of the projector, screen, tilt angle of the windshield, and for the desired eyebox position and size. The HUD design can be customized for the specific applications and arbitrary designs such as different car models.

\section{B. Basic Screen Structure}

In our embedded see-through screen, we have designed an MLA with each microlens rotated about two axes, such that the incident beam is reflected toward the driver's eyes. In other words, the pointing

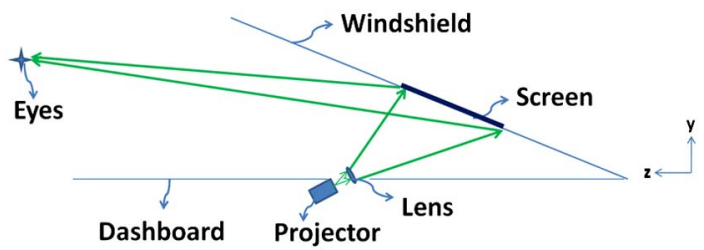

Fig. 4. (Color online) HUD system with the projector on the dashboard. The projection cone can be expanded with a small lens such that the whole screen can be projected with the desired image. of the microlenses steers the light coming from the projector toward the eyes of the driver while the curvature of microlenses expands the incident beam to create an eyebox. As a result, eyeboxes corresponding to every pixel on the screen overlap almost perfectly, so the available light is used more efficiently. This produces a usable eyebox where the individual pixel eyeboxes overlap, solving the problem shown in Fig. 3, and because they overlap completely, it effectively increases the screen gain, giving more brightness than the partially overlapped case achieved by a planar MLA screen as in [5]. Additionally, the tilting of each microlens, based on the specific geometry in a given car, compensates for the angle of the windshield from the position of the projector and therefore provides greater freedom of where to position the projector.

Figure 5 illustrates the structure of the screen. The embedded see-through screen structure is designed to substitute for the polyvinyl butyral layer typically sandwiched between the two glass layers of the windshield to create safety glass. The see-through screen itself is a sandwich structure beginning with a molded MLA that has the desired form of rotated microlenses. Epoxy casting could be used, as shown in Fig. 5 , although other polymer or plastic materials could also be used. The MLA is then coated with a partially reflective thin coating. Either metal or dielectric partial-reflective coatings could be used depending on the desired properties of the screen. Finally, the coated surface is covered with another layer of the same material used under the coating layer so that the partially reflective coating is the only index mismatch in the full sandwich structure. The whole structure is buried between the layers of the windshield. The transmitted beam does not experience any spatially varying phase and propagates

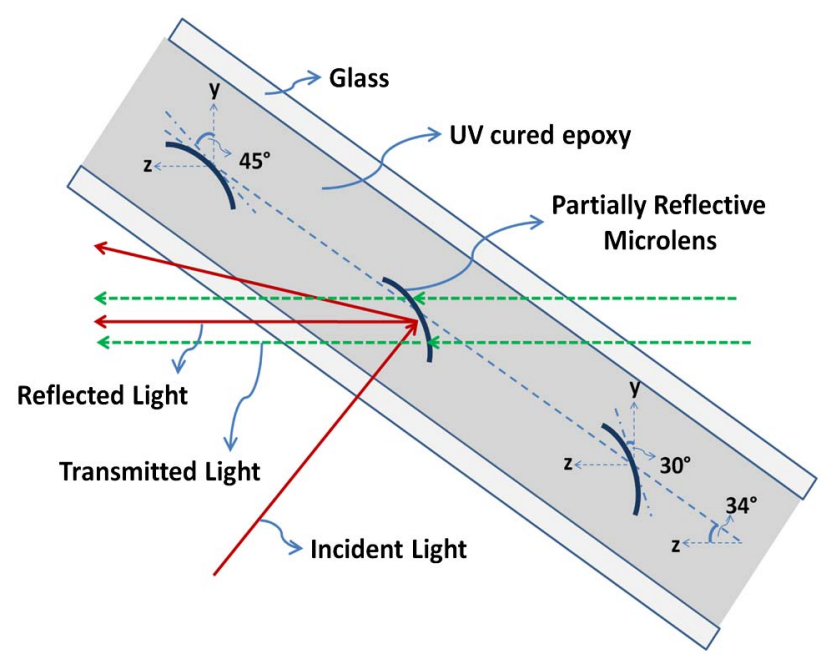

Fig. 5. (Color online) Side view of three sample microlenses across the screen. Each partially reflective coated microlens is rotated individually and sandwiched between the index matching layers of epoxy. The index matched structure does not distort the transmitted light, but the reflected light is expanded by the microlenses toward the eyebox. 
through the glass without any changes to the wavefront. The reflected beam, on the other hand, is directed toward the eyes and forms an expanded eyebox [5].

As the screen has a faceted surface and it is used off-axis, one of the design challenges is to avoid the shadowing effect from adjacent microlenses that blocks the light coming from the lens immediately below. Although the microlenses are $150 \mu \mathrm{m}$ tall, the MLA pitch is kept $300 \mu \mathrm{m}$ constant in both directions, as illustrated in Fig. $\underline{6}$ to avoid shadowing; in other words, the MLA pitch is kept constant, but the aperture size is varied. As discussed in detail in [5], the MLA pitch optimal value is around $300 \mu \mathrm{m}$. The pitch is optimized using two constraints: (i) it should be smaller than the display pixels on the screen and (ii) it should be large enough to keep the diffraction order spacing at the eye smaller than the minimum pupil size to avoid intensity variations as the eye moves within the eyebox.

With the current screen and microlens dimensions, the resolution is approximately $583 \times 292$ pixels (calculated as screen size divided by microlens pitch). The maximum resolution of the laser scanning based picoprojector used in [5] was WVGA $(850 \times 480)$ pixels. The additional resolution of the projector provides overhead for use in electronically correcting for keystone and other distortions.

Partial reflective coatings are important for the operation of the screen. If we assume a broadband, partially reflective coating, a good first-order estimate for the screen properties is governed by the relationship $R+T+A=1$, where $R$ is reflectance, $T$ is transmittance, and $A$ is absorption. A single layer metal coating is the simplest. The amount of transmittance and reflectance varies with the thickness of the metal layer. In our testing, an $80 \AA$ thick $\mathrm{Al}$ coating produced a screen with about $30 \%$ reflectance, but the metal layer also causes absorption, about $30 \%$ in this case, bringing the transmittance down to approximately $40 \%$. With a broadband dielectric coating, absorption is avoided, so for $30 \%$ broadband reflectance, the transmittance is increased to approximately $70 \%$. It is important to get a flat spectral response from the dielectric coating, so that no

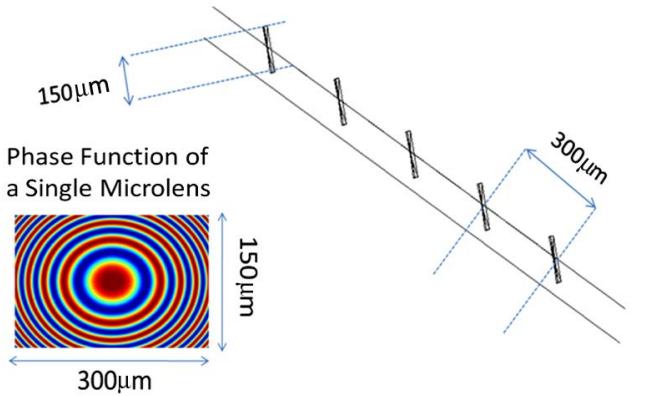

Fig. 6. (Color online) Horizontal cross section of part of the screen in the Zemax model, showing five consecutive microlenses. Microlenses are placed $300 \mu \mathrm{m}$ apart to eliminate shadowing. Spherical microlenses with rectangular apertures are used in the array. coloration is added to the screen. A third option, which may be effective if a laser projector is used, is to apply a notch coating as the partially reflective layer. The notch coating would be designed to produce high reflectance at the laser wavelengths and low reflectance for the rest of the visible spectrum. In this way, the efficiency of relaying the projected light to the driver's eyes can be increased while still maintaining a high average transmittance across the visible band. The effect of coatings on the screen transmission characteristics and the MTF will be discussed in more detail in a future publication.

\section{Calculating the Rotation Angles of the Microlenses}

We calculated the rotation angles of the microlenses using the following methodology: Since the tilting of the microlenses is intended to center the individual pixel eyeboxes between the driver's eyes, we begin by treating the microlenses as planar micromirrors. We then calculate their rotations about the $x$ and $y$ axes to steer the incoming light from the projector toward the driver. Finally, we convert the rotated flat micromirrors into microlenses to expand the light to create the eyebox. The most important part of our design is the calculation of the rotation angles of the microlenses to reflect the incident beam toward the driver's eyes. The rotation angles are calculated based on the positions of the projector, the driver, and the individual microlenses, using the method described below. In this paper, we assumed that the windshield was planar as a representative example of the calculation procedure. Since the microlens rotation angles are calculated based on the position of the individual microlenses, our algorithm can calculate the rotation angles based on the surface profile of any particular windshield.

Since the microlenses are buried in an indexmatched layer, the incident and reflected light are subject to refraction due to the refractive index difference between the windshield and the surrounding air, as illustrated in Fig. 7(a). Thus, the problem of finding the aiming point on the interface to get the light crossing the desired point in the other medium must be included in the calculations. Figure 7(b) illustrates the details of refraction at the glass interface. Equations below are used to calculate the vectors $\mathbf{V}_{\mathrm{i} 1}$ and $\mathbf{V}_{r 1}$ to find the path from projector to micromirror. The path from the micromirror to the driver's eye is calculated in a similar manner by applying the same set of equations to find $\mathbf{V}_{\mathbf{i} 2}$ and $\mathbf{V}_{r 2}$ in Fig. 7(a).

Snell's law in vector form is shown in Eq. (1), where $\mathbf{n}$ is the unit surface normal vector of the interface, $\eta$ is the ratio of the refractive indices $n_{i} / n_{r}$, and $\mathbf{v}_{\mathbf{i} 1}$ and $\mathbf{v}_{\mathbf{r} 1}$ are the unit vectors along the incident and refracted light, respectively [7]. As both the incident and refracted vectors are not known, a second equation is needed to obtain two equations with two unknowns. A weighted sum of $\mathbf{v}_{\mathbf{i} 1}$ and $\mathbf{v}_{\mathbf{r} 1}$ should result in the desired vector $\mathbf{v}_{\mathbf{d}}$, which is the vector between the desired initial and final points, as illustrated in 


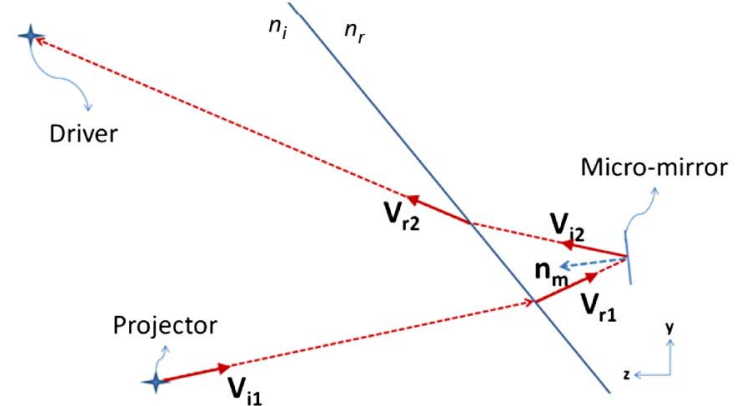

(a)

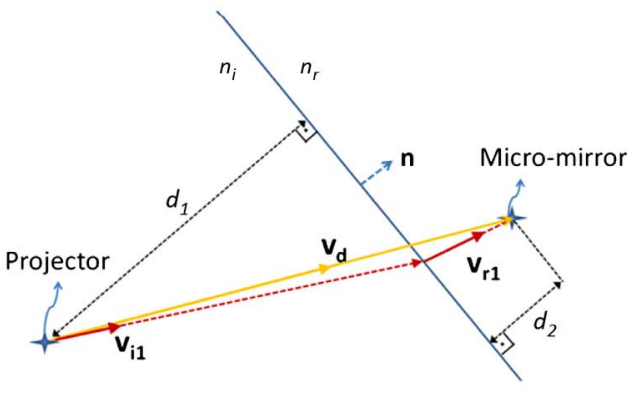

(b)

Fig. 7. (Color online) (a) Because of the screen structure the incident and reflected light are subject to refraction. Based on the incident and reflected vectors on the micromirror, the surface normal is calculated. (b) The aiming point on the windshield should be calculated such that the refracted light passes through the desired point on the other side. The procedure of finding the incident and refracted vectors $\mathbf{v}_{\mathbf{i} 1}$ and $\mathbf{v}_{\mathbf{r} 1}$ is followed twice for each micromirror, from projector to the micromirror and from micro-mirror to the driver.

Fig. $7(\mathrm{~b})$. The weights of the vectors should be selected as in Eq. (2), where $d_{1}$ and $d_{2}$ are the distances of the initial and final points to the interface plane and $\left(\mathbf{v}_{\mathbf{i} 1} \cdot \mathbf{n}\right)$ and $\left(\mathbf{v}_{\mathbf{r} 1} \cdot \mathbf{n}\right)$ are the dot products of the incident and refracted unit vectors with the interface surface normal, respectively. Equation (3) is obtained by solving Eq. (1) and Eq. (2) together, where $\left(\mathbf{v}_{\mathbf{i} 1} \cdot \mathbf{n}\right)$ is the only independent unknown. As the dot product is a scalar quantity, the incident vector $\mathbf{v}_{\mathbf{i} 1}$ is expressed as a single variable function of $\left(\mathbf{v}_{\mathbf{i} 1} \cdot \mathbf{n}\right)$ We know that $\mathbf{v}_{\mathbf{i} 1}$ is a unit vector so its norm should be equal to 1 . To get the correct value of $\left(\mathbf{v}_{\mathbf{i} 1} \cdot \mathbf{n}\right), f(x)$ in Eq. (4) is minimized iteratively using the NewtonRaphson method []], where $x$ denotes $\left(\mathbf{v}_{\mathbf{i} 1} \cdot \mathbf{n}\right)$ :

$$
\begin{aligned}
\mathbf{v}_{\mathbf{r} 1}= & \left(\operatorname{sign}\left(\mathbf{v}_{\mathbf{i} 1} \cdot \mathbf{n}\right) \sqrt{1-\eta^{2}+\eta^{2}\left(\mathbf{v}_{\mathbf{i} 1} \cdot \mathbf{n}\right)^{2}}\right. \\
& \left.-\left(\mathbf{v}_{\mathbf{i} 1} \cdot \mathbf{n}\right) \eta\right) \mathbf{n}+\eta \mathbf{v}_{\mathbf{i} 1} \\
\mathbf{v}_{\mathbf{d}}= & \frac{d_{1}}{\mathbf{v}_{\mathbf{i} 1} \cdot \mathbf{n}} \mathbf{v}_{\mathbf{i} 1}+\frac{d_{2}}{\mathbf{v}_{\mathbf{r} 1} \cdot \mathbf{n}} \mathbf{v}_{\mathbf{r} 1}, \\
\mathbf{v}_{\mathbf{i} 1}= & \frac{\mathbf{v}_{\mathbf{d}}-d_{2}\left(1-\frac{\eta\left(\mathbf{v}_{\mathbf{i}} \cdot \mathbf{n}\right)}{\sqrt{1-\eta^{2}+\eta^{2}\left(\mathbf{v}_{\mathbf{i} 1} \cdot \mathbf{n}\right)^{2}}}\right) \mathbf{n}}{\frac{d_{1}}{\left(\mathbf{v}_{\mathbf{i} 1} \cdot \mathbf{n}\right)}+\frac{\eta d_{2}}{\sqrt{1-\eta^{2}+\eta^{2}\left(\mathbf{v}_{\mathbf{i} 1} \cdot \mathbf{n}\right)^{2}}}} \\
f(x)= & \left\|\mathbf{v}_{\mathbf{i} 1}\right\|-1 .
\end{aligned}
$$

Once $\mathbf{v}_{\mathbf{i} 1}$ is obtained by plugging in the computed value of $\left(\mathbf{v}_{\mathbf{i} 1} \cdot \mathbf{n}\right)$ in Eq. ( $\left.\underline{3}\right), \mathbf{v}_{\mathbf{r} 1}$ can be calculated using Eq. (1). This procedure is followed two times for each micromirror: for finding the unit incident and refracted vectors from the projector to the micromirror and from the micromirror to the driver, as shown in Fig. 7(a) as $\mathbf{V}_{\mathbf{i} 1}, \mathbf{V}_{\mathbf{r} 1}, \mathbf{V}_{\mathbf{i} 2}, \mathbf{V}_{\mathbf{r} 2}$, respectively. Surface normal of the micro-mirror should be calculated such that when $\mathbf{V}_{\mathbf{r} 1}$ is the incident unit vector, $\mathbf{V}_{\mathbf{i} 2}$ should be the reflected unit vector. Equation (ㅁ) gives the reflected unit vector $\mathbf{V}_{\mathrm{i} 2}$, when a unit vector $\mathbf{V}_{\mathbf{r} 1}$ is incident on a surface with surface normal $\mathbf{n}_{\mathbf{m}}$ [7]. In our case, we know the incident and reflected vectors and we need the surface normal vector. Using the fact that angle of incidence is equal to the angle of reflection, Eq. (ㅁ) can be transformed into Eq. (6), which gives the surface normal when incident and reflected vectors are known [9].

After we find the surface normal vector, the required rotation angles can be calculated by solving the rotation matrix shown in Eq. (7), where $\theta$ and $\varphi$ are the rotations about the $x$ and $y$ axes, respectively. $x_{m}, y_{m}, z_{m}$ in Eq. (7) are the components of the vector $\mathbf{n}_{\mathbf{m}}$. Unrotated micromirrors are assumed to have unit surface normal vectors parallel to the $z$ axis. [The coordinate axes are shown in Fig. (느).]

$$
\begin{aligned}
& \mathbf{v}_{\mathbf{i} 2}=\mathbf{v}_{\mathbf{r} 1}-2\left(\mathbf{v}_{\mathbf{r} 1} \cdot \mathbf{n}_{\mathbf{m}}\right) \mathbf{n}_{\mathbf{m}}, \\
& \mathbf{n}_{\mathbf{m}}=\frac{\mathbf{v}_{\mathbf{r} 1}-\mathbf{v}_{\mathbf{i} 2}}{\sqrt{2\left(1-\left(\mathbf{v}_{\mathbf{i} 2} \cdot \mathbf{v}_{\mathbf{r} 1}\right)\right)}},
\end{aligned}
$$

$\left[\begin{array}{ccc}\cos \varphi & 0 & \sin \varphi \\ \sin \varphi \sin \theta & \cos \theta & -\cos \varphi \sin \theta \\ -\sin \varphi \cos \theta & \sin \theta & \cos \varphi \cos \theta\end{array}\right]\left[\begin{array}{l}0 \\ 0 \\ 1\end{array}\right]=\left[\begin{array}{l}x_{m} \\ y_{m} \\ z_{m}\end{array}\right]$,

$$
\begin{aligned}
& \varphi=\sin ^{-1}\left(x_{m}\right), \\
& \theta=\tan ^{-1}\left(-\frac{y_{m}}{z_{m}}\right) .
\end{aligned}
$$

MATLAB was used to calculate the rotation angles for each micromirror on the screen using the method described above. Figure 8 shows the $2 \mathrm{D}$ contour plot of the magnitude of the compound rotation angles for the design in Fig. 4 as a function of micromirror position, that is, $\left[\theta^{2}+\bar{\varphi}^{2}\right]^{1 / 2}$. The tilt direction is normal to the contour lines. 


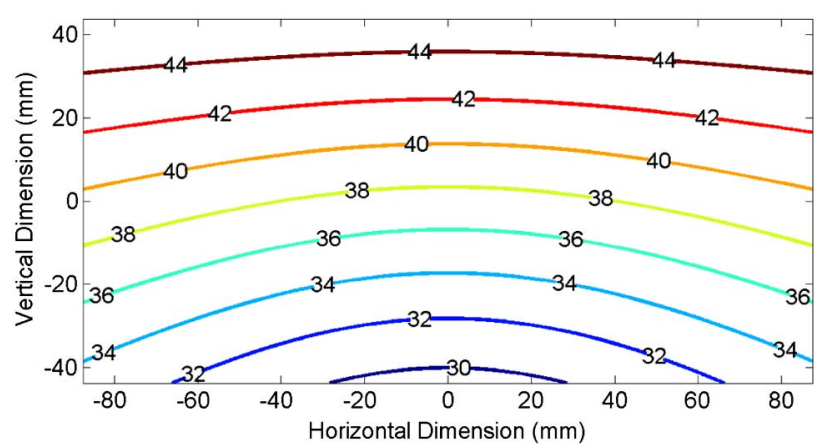

Fig. 8. (Color online) Contour plot of the calculated rotation angles of the whole screen in degrees. The tilt direction is normal to the contour lines.

\section{Simulations}

We used five sample points on the screen to simulate the system in Zemax, one from each corner and one from the center. The side view of the system is shown in Fig. 9 , which shows the focusing characteristic of our screen with flat micromirrors. Once the flat micromirrors are converted into microlenses, the resulting eyeboxes for each point on the screen overlap almost perfectly. This maximizes the gain of the screen by steering most of the light to the fully overlapped region of the eyeboxes, where the complete field of view can be seen.

We used a $635 \mathrm{~nm}$, collimated Gaussian beam as the source in the Zemax model for simulating the screen. In Fig. 10, the eyeboxes for the five selected points are shown at the driver's position. There is some geometric distortion due to the off-axis operation of the microlenses, which results in an elongation of the eyebox. The chromatic aberrations due to inclined transmission in glass have been checked in the Zemax model and found to be negligible.

The gain of the MLA screen, which is the ratio of the intensity compared to that obtained with a Lambertian scatterer, is calculated as 69 . The perceived brightness within the eyebox using a 20 lumen projector is $7171 \mathrm{~cd} / \mathrm{m}^{2}$, assuming $50 \%$ reflective screen. This exceptional brightness can be utilized in two different ways: The projector power can be lowered and consequently the energy dissipation of the whole system is lowered, or the reflectivity of the

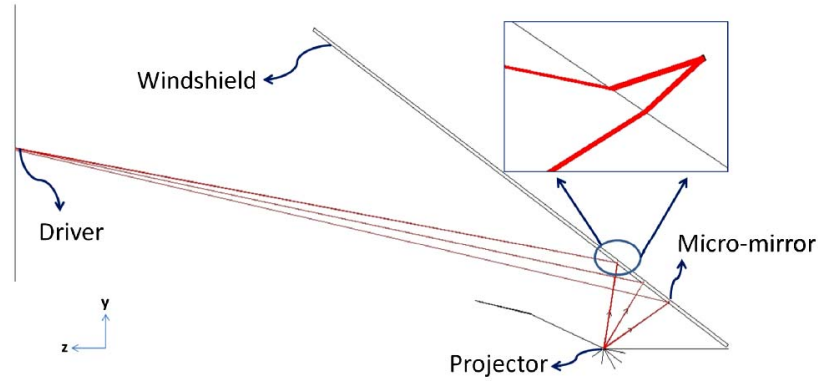

Fig. 9. (Color online) Side view of the system, showing the focusing characteristic of our screen due to the rotated micro-mirrors used in the design.

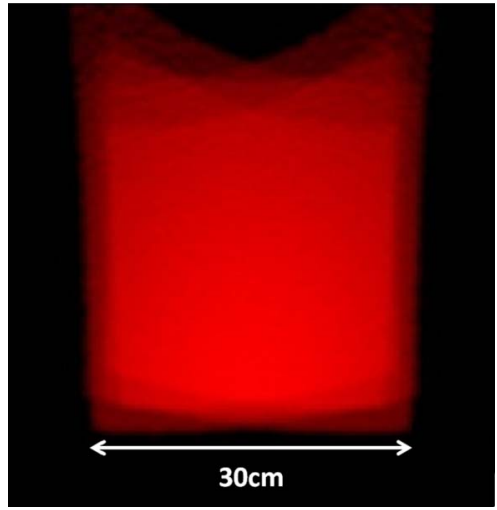

Fig. 10. (Color online) True color image of the eyeboxes at the driver's position, corresponding to the same sample points in Fig. 3 . They overlap very well thanks to the rotated microlenses.

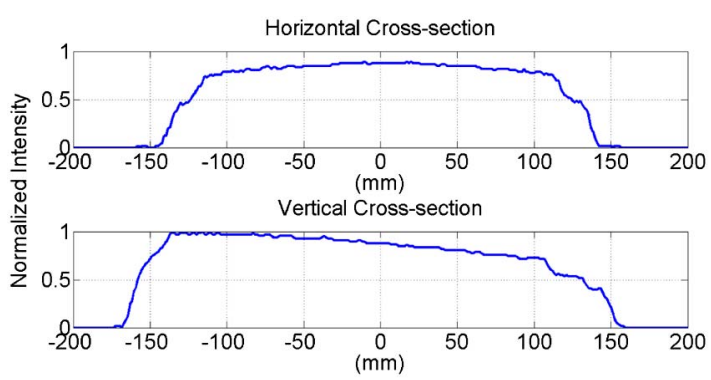

Fig. 11. (Color online) Horizontal cross section of the normalized eyebox intensity on top and the vertical cross section at the bottom. The horizontal uniformity is very good so the eyes perceive the same brightness.

screen can be decreased and hence the transparency is enhanced, making it even more difficult to distinguish the screen from the windshield.

The design target for the eyebox width is $250 \mathrm{~mm}$. The horizontal and vertical cross sections of the simulated eyebox are shown in Fig. 11. As the eyes are oriented horizontally, the variations in the horizontal cross section are perceived more easily. The horizontal cross section of the eyebox is quite uniform, so that the perceived brightness is almost equal for both eyes and it is sufficiently large for comfortable operation. The vertical cross section shows the margin of the system to accommodate different heights of the drivers.

\section{Conclusion}

HUD systems are becoming a standard feature in cars due to the improved safety they provide. Presenting the necessary information to drivers without taking their eyes from the road improves the response time of the drivers. The embedded direct projection HUD system we have presented here can provide a wide field-of-view HUD without taking up a lot of space within the car. This paper has focused on the design of a see-through screen that can be embedded into the windshield.

We have presented a general design methodology for the embedded see-through screen that can be 
applied to create screen designs for any specific car and arbitrary car layouts, as well as accommodate arbitrary FOVs and eyebox sizes. It has the property that the light from every pixel is overlapped at the driver's eye position, thereby using the projected light efficiently and making it possible to produce a very bright display with a low power (only 20 lumen) picoprojector that can be viewed from an eyebox area of about $30 \mathrm{~cm} \times 30 \mathrm{~cm}$.

Custom MLA fabrication with varying tilts across a large area is challenging and requires an expensive large area master. Standard step-and-repeat procedures using a small master cannot be used here, as each part of the screen is different. The master can be produced with high-precision diamond cutting or laser writing technologies. However, once the master is made, replication should be relatively straightforward using standard molding technologies.

We would like to thank and acknowledge Microvision Inc. for their support in this research. Mark Freeman, the second author of this paper, was the director of Advanced Development and HUD at Microvision when this research was performed.

\section{References}

1. Y.-C. Liu and M.-H. Wen, "Comparison of head-up display (HUD) vs. head-down display (HDD): driving performance of commercial vehicle operators in Taiwan," Int. J. HumanComputer Studies 61, 679 (2004).

2. N. A. Kaptein, "Benefits of in-car head-up displays," TNO Report TNO-TM 1994, B-20, TNO Human Factors Research Institute (1994).

3. M. Ablassmeier, T. Poitschke, and G. Rigoll, "Eye gaze studies comparing head-up and head-down displays in vehicles," in Proceedings of IEEE Conference on International Conference on Multimedia and Expo (IEEE, 2007), pp. 2250-2252.

4. M. O. Freeman, "MEMS scanned laser head-up display," Proc. SPIE 7930, 79300G (2011).

5. M. K. Hedili, M. O. Freeman, and H. Urey, "Microstructured head-up display screen for automotive applications," Proc. SPIE 8428, 84280X (2012).

6. H. Urey and K. D. Powell, "Microlens-array-based exit-pupil expander for full-color displays," Appl. Opt. 44, 4930-4936 (2005).

7. D. Fontijne and L. Dorst, "Modeling 3D Euclidean geometry," IEEE Comput. Graph. Appl. 23, 68-78 (2003).

8. J. E. Dennis and R. B. Schnabel, Numerical Methods for Unconstrained Optimization and Nonlinear Equations (PrenticeHall, 1983), Chap. 2.

9. F. L. Pedrotti, L. M. Pedrotti, and L. S. Pedrotti, Introduction to Optics, 3rd ed. (Pearson Prentice-Hall, 2007). 